\title{
Study of Thiol Protected Au/Co Nanoclusters Using Aberration Corrected STEM
}

\author{
N. Bhattarai, D. Bahena, S. Khanal, A. Ponce and M. Jose-Yacaman \\ Department of Physics and Astronomy, University of Texas at San Antonio, One UTSA Circle, San \\ Antonio, Texas 78249, USA.
}

The study of magnetic bimetallic nanocluster with plasmonic properties is very fascinating area of nanoscience because of the presence of both plasmonic and magnetic properties. They are widely used such as: biomedicine, catalysis, waste water management, data storage, drug delivery, and many more [1-3]. The synthesis of small magnetic nanoclusters of defined sizes is very challenging because of agglomeration, nonuniform size, shape and narrow size distributions. Here in, we report the fabrication of 3-5 nm sized monodispersed dodecanethiol protected $\mathrm{Au} / \mathrm{Co}$ alloyed nanoclusters by using modified Brust's method and its atomistic investigations using aberration corrected scanning/transmission electron microscopy (STEM)[4].

During the imaging of thiolated NCs, the cleanliness of the sample, operating voltage of the microscope and exposure time of beam to the sample plays significant role in order to get atomically resolved images without destroying the particle shape and attached ligands. Low magnification STEM images, size distribution and some typical HAADF STEM images of $\mathrm{Au} / \mathrm{Co} \mathrm{NC}$ is presented in Fig. 1. Highly monodispersed 3 to $5 \mathrm{~nm}$ sized nanoclusters with decahedral and icosahedral shape are observed. The Z-contrast image shows the atomic positions of $\mathrm{Au}$ (strong contrast) and Co (weak contrast) as presented in Fig. 2a. The colored image of squared portion of Fig. 2a in fig. $2 \mathrm{c}$ shows the atomic positions of Au with orange spots and Co with white circled spots. Moreover, the intensity profile along the arrowed direction in Fig. 2a is presented in Fig. 2d, where we can see the intensity contributions from $\mathrm{Au}$ and Co again confirming alloying. Furthermore, the distribution of Au and Co is observed by EDS line scan as presented in Fig. 3a and b. The overlapping and crossing of the spectrum showed the alloyed $\mathrm{Au} / \mathrm{Co}$ nanostructure. In conclusion, we are able to fabricate 3-5 nm sized dodecanethiol protected $\mathrm{Au} / \mathrm{Co}$ nanocluster and able to prove the alloying between them at nanoscale.

\section{References:}

[1]Reiss, G.; Hutten, A., Nature Materials 2005, 4, 725.

[2]Moghimi, S.M., A.C. Hunter, and J.C. Murray, Pharmacological Reviews, 2001, 5, 283.

[3]Mayoral, A.; Mejia-Rosales, S.; Mariscal, M. M.; Perez-Tijerina, E.; Jose-Yacaman, M., Nanoscale 2010, 2, 2647.

[4]Brust, M.; Schiffrin, D. J.; Bethell, D.; Kiely, C. J., Advanced materials 1995, 7, 795.

[5]Acknowledgments: The authors would like to acknowledge NSF grants DMR-1103730, "Alloys at the Nanoscale: The Case of Nanoparticles Second Phase" and PREM: NSF PREM Grant \# DMR 0934218. 

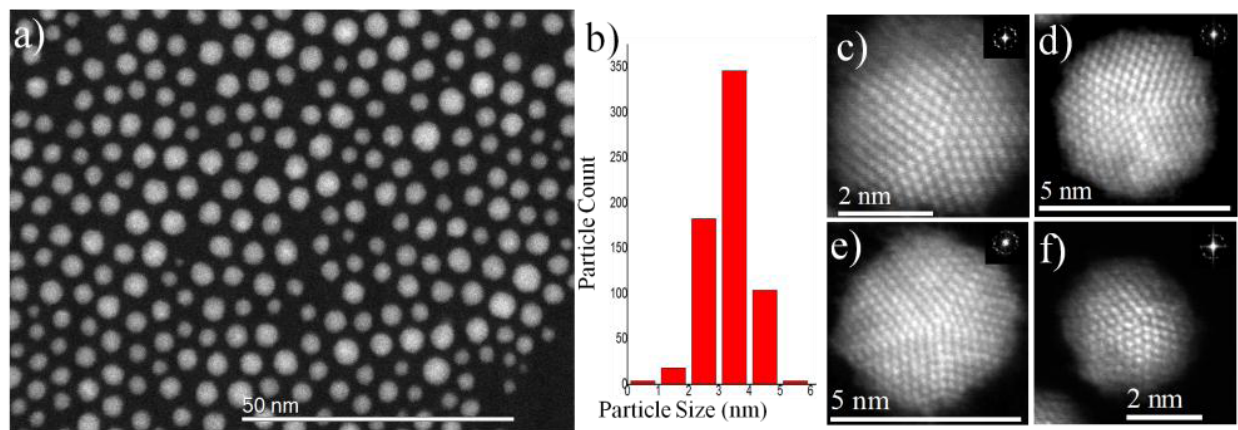

Fig. 1. a) and b) Low magnification STEM image of 3 to $5 \mathrm{~nm}$ sized monodispersed $\mathrm{Au} / \mathrm{Co}$ nanoclusters and its histogram. c)-f) HAADF STEM images of typical decahedral (c-e) and icosahedral (f) nanoclusters with FFT patterns inset.
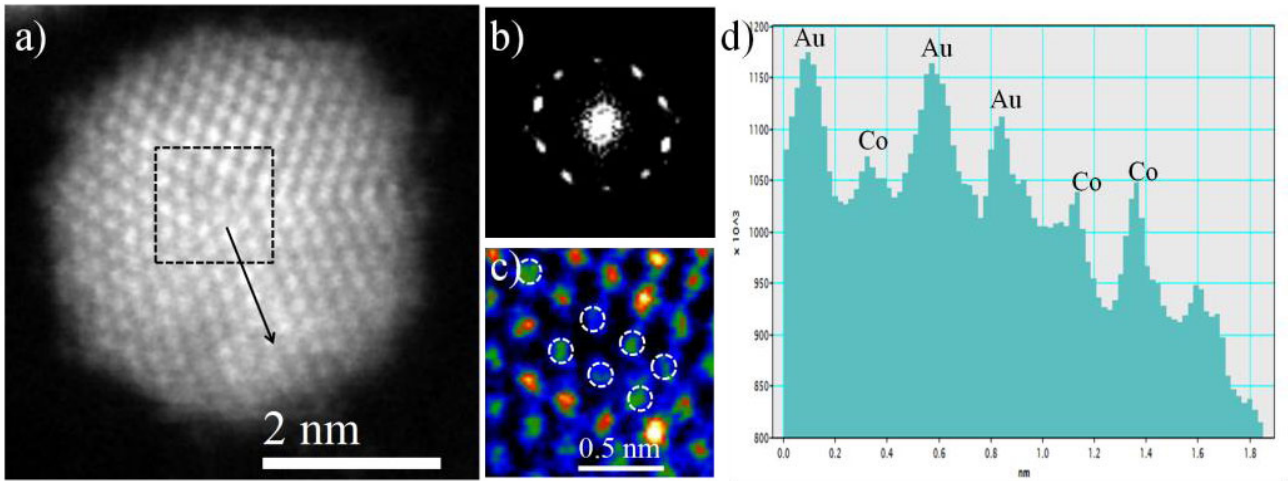

Fig. 2. a) A representative decahedral STEM image. b) and c) FFT pattern in a and colored image of the squared region in a where the clear difference in the intensity of points gives the distribution of $\mathrm{Au}$ (orange) and Co (white circled) in the nanostructure. d) The intensity profile along the arrowhead with the positions of $\mathrm{Au}$ and $\mathrm{Co}$.
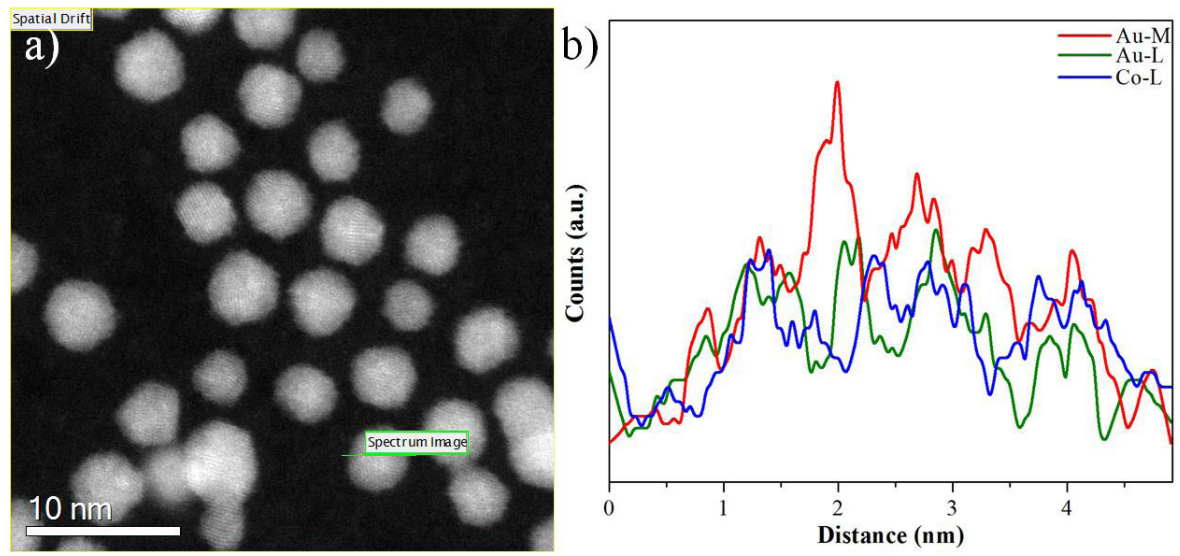

Fig. 3. EDS line profile of single $\mathrm{Au} / \mathrm{Co}$ nanocluster. a) Dark field images with the spatial drift and spectrum image during recording EDS spectra. b) The line profile spectrum obtained from EDS analysis. 